www.jmscr.igmpublication.org

Impact Factor (SJIF): 6.379

Index Copernicus Value: 71.58

ISSN (e)-2347-176x ISSN (p) 2455-0450

crossref DOI: _https://dx.doi.org/10.18535/jmscr/v6i3.157

Journal Of Medical Science And Clinical Research

IGM Publication

An Official Publication of IGM Publication

\title{
Clinical Profile of Children with Severe acute Malnutrition Admitted to the Nutritional Rehabilitation Centre of a Tertiary Care Teaching Hospital of Assam
}

\author{
Authors \\ Manab Narayan Baruah ${ }^{1}$, Bhaskarjit Gogoi ${ }^{2}$, Pranabjit Biswanath ${ }^{3}$ \\ ${ }^{1}$ Assistant Professor, ${ }^{2}$ Dietician, ${ }^{3}$ Head of Department \\ ${ }^{1,2,3}$ Department of Pediatrics, Jorhat Medical College, Jorhat, Assam, India-785001 \\ Corresponding Author \\ Manab Narayan Baruah \\ Department of Pediatrics, Jorhat Medical College, Jorhat, Assam, India-785001 \\ Email:drmnbaruah@gmail.com
}

\begin{abstract}
Background: Severe acute malnutrition (SAM) is the main contributing factor for under-five morbidity and mortality. Nutritional Rehabilitation Centre (NRC) provides a protocol based management of SAM based on the guidelines laid down by the World Health Organization (WHO). The NRC model not only envisages the short term aim to reduce mortality due to SAM but with a community linkage and effective follow up, it is aimed at a comprehensive and long term improvement in quality of life in undernourished children. The NRC at Jorhat Medical College can serve as a model for hospital based management of SAM in our state.

Aims of the Study: To study the clinical profile of children with severe acute malnutrition admitted to the Nutritional Rehabilitation Centre of a teaching hospital of upper Assam.

Materials and Methods: Retrospective study based on review of case records of children between age group of 6months up to 5 years with Severe Acute Malnutrition (SAM) admitted in NRC, Department of Pediatrics, Jorhat Medical College between $1^{\text {st }}$ April 2016 to $31^{\text {st }}$ March 2017.

Results: Out of the total of 150 SAM children admitted in the NRC, 128(85.3\%) were from the tea garden community. Majority of SAM $(n=52,34.7 \%)$ presented in the second year of life. The mode of referral of cases to NRC was by the physician ( $n=59,39.3 \%)$ and Anganwadi worker $(n=42,28 \%)$ followed by self referral, ASHA worker and others like NGOs. Fever, cough, feeding problems and loose motion were the chief presenting complaints; Urinary tract infection was the most common co-morbidity (22.7\%). The mean duration of stay was 10 days. $13.3 \%$ of cases showed satisfactory weight gain of more than $10 \mathrm{gm} / \mathrm{kg} / \mathrm{day}$ while majority had moderate weight gain.

Conclusion: Hospital based management of SAM children in NRC is a significant step in reducing morbidity and mortality among undernourished children. However, there is a need to improve the quality of care and ensure satisfactory follow up with active involvement of the community stake holders.
\end{abstract}

Keywords: Nutritional Rehabilitation Centre, SAM, weight for height, MUAC.

\section{Introduction}

Globally, malnutrition is responsible for almost one-half $(45 \%)$ of the total under five deaths. Besides increased mortality, malnutrition is associated with increased morbidity in terms of recurrent infections, growth retardation, and impaired long term psychosocial and cognitive development ${ }^{1}$. India being home to eight million 
children with severe acute malnutrition accounts for $20 \%$ of the deaths annually. The NFHS-3 data shows that $43 \%$ of our children are underweight, $48 \%$ are stunted, $20 \%$ are wasted with $7.9 \%$ being severely wasted ${ }^{2}$. The late introduction, inadequate quantity and concurrent infections are responsible for rise in under nutrition at 611 months age which continues in the second year of life $\mathrm{e}^{3}$.

The initial crusade against SAM by WHO (World Health Organization) was through formulation of treatment guidelines for physicians and health workers in 1999 which was revised in the year 2002. Children with weight for height $<-3$ SD have a nine fold higher risk of death compared to those with parameter above -1SD.The Indian Academy of Pediatrics (IAP) in 2006 adopted the WHO guidelines for hospitalised management of severely malnourished children. The protocol based in patient management of SAM has witnessed a significant reduction in case fatality rate from $40 \%$ to $20 \%{ }^{5}$.

The establishment of Nutritional Rehabilitation Centre (NRC) as an inpatient protocol based centre for management of SAM is an important step by the National Health Mission. The first NRC was set up in Madhya Pradesh in 2007 followed by many states including Assam ${ }^{6}$. Jorhat Medical College and Hospital (JMCH) is the only medical college in Assam to have a NRC. JMCH caters not only to the population of Jorhat but also the neighbouring districts of Golaghat and Sivasagar -all with a sizeable tea garden population where under nutrition is common.

There is paucity of data regarding the functioning of NRC in Assam and the north eastern India. So, the present study was undertaken to study the clinical profile of SAM children admitted to the Nutritional Rehabilitation Centre (NRC) of Jorhat Medical College- a tertiary care teaching hospital of upper Assam.

\section{Aim of the Study}

To study the clinical profile of children with severe acute malnutrition admitted to the
Nutritional Rehabilitation Centre of a teaching hospital of upper Assam.

\section{Materials and Methods}

Study Design: Retrospective study based on review of case records of patients admitted in NRC, Department of Pediatrics, JMCH.

Study population: All SAM children admitted to NRC of JMCH between $1^{\text {st }}$ April 2016 to $31^{\text {st }}$ March 2017.

Study period: 1 year.

Inclusion criteria: Children between the ages of 6months up to 5years who fulfilled the definition of SAM by WHO were included in the study. SAM is defined as the presence of any one of the following features (in the age group 6months- 59 months).

1. Weight for height $<-3 \mathrm{SD}$

2. Mid upper arm circumference $($ MUAC) < $11.5 \mathrm{~cm}$

3. Visible severe wasting

4. Bipedal oedema.

Exclusion criteria: Children with non nutritional cause for underweight, chronic disorders like cardiac, renal, CNS or metabolic disorders were excluded from the study.

In all admitted patients, a detailed history and physical examination were done. Anthropometric indices like weight, length/height, MUAC and weight for height were recorded and analysed using WHO growth charts. Investigations including complete hemogram, microscopy for malaria, urine routine and culture, CXR, mantoux test, blood sugar and electrolytes were done in all cases. All patients were started initially on F-75 diet along with calorie dense locally available food which is prepared in the NRC kitchen. The mothers are made to participate actively in the preparation and serving of food to their children.

\section{Results and Observations}

During the 1 year study period, a total of 150 SAM children were admitted to NRC, JMCH out of which 68 (45.3\%) were males and 82(54.7\%) were females (Table no 1). There is a 
preponderance of SAM children from the tea garden community $(n=128,85.3 \%)$ in our study.

Table no 1: Showing the gender distribution of SAM cases

\begin{tabular}{|l|c|}
\hline Total & $\mathrm{n}=150$ \\
\hline Male & $68(45.3 \%)$ \\
\hline Female & $82(54.7 \%)$ \\
\hline
\end{tabular}

The age wise distribution (Table no 2) shows that maximum no of cases were in the second year of life $(n=52,34.7 \%)$. The first 2 years of life contributed to half of all cases $(n=78,52 \%)$.

Table no 2: Age wise distribution of admitted cases

\begin{tabular}{|l|c|}
\hline Age at admission & No of cases \\
\hline 6months- 12 months & $\mathrm{n}=26(17.3 \%)$ \\
\hline 1-2 years & $\mathrm{n}=52(34.7 \%)$ \\
\hline 2-3 years & $\mathrm{n}=25(16.7 \%)$ \\
\hline 3-4 years & $\mathrm{n}=15(10 \%)$ \\
\hline 4- 5 years & $\mathrm{n}=32(21.3 \%)$ \\
\hline
\end{tabular}

Figure no 1: Ethnic distribution of SAM cases

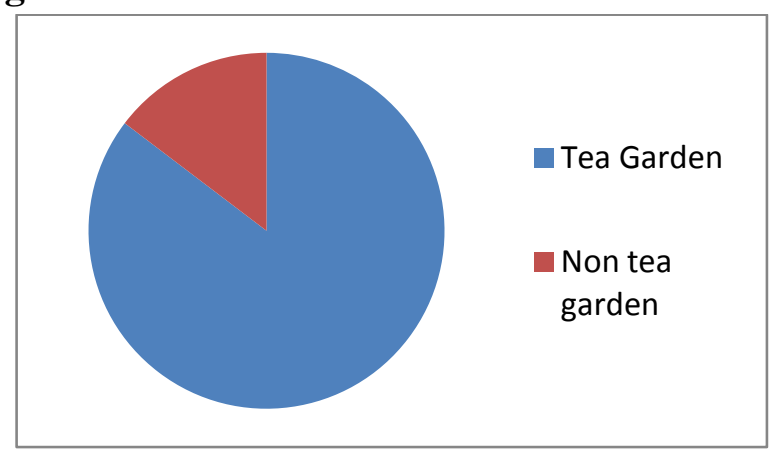

The early identification of SAM cases in the community and prompt referral is an important aspect of SAM management and success of NRC. The present study tried to analyse the mode of referral as shown in Table no 3.

Table no 3: Showing mode of referral of cases to NRC.

\begin{tabular}{|l|c|}
\hline ASHA & 12 \\
\hline Anganwadi worker & 42 \\
\hline Physician & 59 \\
\hline Nutrition counsellor & 7 \\
\hline Self & 18 \\
\hline Others & 12 \\
\hline
\end{tabular}

The above data shows that majority of cases were referred to NRC by the physician $(n=59,39.3 \%)$ and Anganwadi worker $(n=42,28 \%)$ followed by self referral, ASHA worker and others like NGOs.
Fever, cough, feeding problems and loose motion were the chief presenting complaints. The present study shows a high incidence of urinary tract infection among SAM cases (Table no 4)

Table no 4: Showing presenting complaints/co morbidities

\begin{tabular}{|l|c|}
\hline Fever & $\mathrm{n}=31(20.7 \%)$ \\
\hline Decreased feeding/refusal to feed & $\mathrm{n}=26(17.3 \%)$ \\
\hline Cough/ Respiratory distress & $\mathrm{n}=26(17.3 \%)$ \\
\hline Diarrhoea & $\mathrm{n}=17(11.3 \%)$ \\
\hline Tuberculosis & $\mathrm{n}=4(2.7 \%)$ \\
\hline Skin infections & $\mathrm{n}=12(8 \%)$ \\
\hline Urinary tract infections & $\mathrm{n}=34(22.7 \%)$ \\
\hline
\end{tabular}

The analysis of duration of stay shows that majority $(97.3 \%)$ were treated for duration of 1-2 weeks with a mean duration of stay of 10 days (Table no 5)

Table no 5: Showing the duration of stay.

\begin{tabular}{|l|c|}
\hline Duration in weeks & No of cases \\
\hline $1-2$ weeks & $\mathrm{n}=146(97.3 \%)$ \\
\hline $2-4$ Weeks & $\mathrm{n}=4(2.7 \%)$ \\
\hline$>4$ weeks & 0 \\
\hline
\end{tabular}

The analysis of weight for height on admission showed that $63.3 \%$ of cases had Z score of <-3SD while $26.7 \%$ had a $Z$ score of $<-4$ SD.

Table no 6: Showing the distribution of weight/height on admission

\begin{tabular}{|l|c|}
\hline$Z$ score & No of cases \\
\hline$<-2$ SD & $n=15(10 \%)$ \\
\hline$<-3$ SD & $n=95(63.3 \%)$ \\
\hline$<-4$ SD & $n=40(26.7 \%)$ \\
\hline
\end{tabular}

$60.7 \%$ of cases fulfilled the WHO MUAC cut off of less than $11.5 \mathrm{~cm}$ as shown in table no 7 .

Table no 7: Showing MUAC at admission

\begin{tabular}{|l|l|}
\hline$<11.5 \mathrm{~cm}$ & $\mathrm{n}=91(60.7 \%)$ \\
\hline $11.5 \mathrm{~cm}-12.5 \mathrm{~cm}$ & $\mathrm{n}=59(39.3 \%)$ \\
\hline
\end{tabular}

The present study shows that $13.3 \%$ of cases had good weight gain while majority $(78.7 \%)$ had moderate rate of weight gain. $8 \%$ of cases had poor weight gain(Table no 8).

Table no 8: Showing the rate of weight gain

\begin{tabular}{|l|c|}
\hline $\mathrm{Gm} / \mathrm{kg} /$ day & No of cases \\
\hline$<5 \mathrm{gm} / \mathrm{kg} /$ day & $\mathrm{n}=12(8 \%)$ \\
\hline $5-10 \mathrm{gm} / \mathrm{kg} /$ day & $\mathrm{n}=118(78.7 \%)$ \\
\hline$>10 \mathrm{gm} / \mathrm{kg} /$ day & $\mathrm{n}=20(13.3 \%)$ \\
\hline
\end{tabular}




\section{Discussion}

A male preponderance was reported by various studies - $74.6 \%$ by Choudhury $\mathrm{M}$ et al (Rajasthan) $^{7}, 53.7 \%$ by Ashraf et $\mathrm{al}^{8}, 55.5 \%$ by Aneja et al ${ }^{9}$ from Maharashtra while others have reported an increased female incidence-78\% Joshi et al.$^{10}$ The present study also shows a higher female admission(54.7\%) with predominance of tea garden community $(85.3 \%)$.

The majority of cases in this study were referred by physician (39.3\%) followed by Anganwadi workers (28\%). B Kumar et al from Madhya Pradesh reported $93 \%$ referral by Anganwadi workers while only $4.8 \%$ were referred by physicians working in the government health facility. So there is a need to further strengthen the process of identification of SAM cases by the community level workers in Jorhat district.

In a study done by Bharti Bhandari and Anita Mehta et al from Gorakhpur ${ }^{11}$, the maximum number of cases was between 1-2 years $(35.03 \%)$ and 2-3 years age groups $(35.03 \%)$. The present study also shows that second year of life had the most no. of cases ( $n=52,34.7 \%)$.

The most common presenting complaint on admission was fever (65\%) and diarrhoea (40\%) in a study done by Rinki Shah et al in Baroda, Gujrat $^{12}$. The present study also shows that fever, cough and diarrhoea as the main presenting complaints. The common co-morbidities associated with SAM cases were gastrointestinal $(60 \%)$ followed by respiratory $(52 \%)$, urinary tract infection (4\%), tuberculosis $(9.3 \%)$, malaria $(1.3 \%)$, otitis media $(2.7 \%)$ as per study done by Choudhury et al in Rajasthan. Bernal et al from Columbia reported $68.4 \%$ incidence of diarrhoea among admitted cases ${ }^{13}$. The present study shows a very high no of cases $(n=34,22.7 \%)$ with urinary tract infections while the incidence of tuberculosis is lower $(2.7 \%)$ compared to that reported by Choudhury from Rajasthan.

The mean length of stay in NRC was 12 days in the study by Manish Maurya et al in Allahabad, $\mathrm{UP}^{14}$ while K Sarala Kumari et al from Telengana reported a mean stay of 11 days with $60 \%$ of parents opting for premature discharge from the $\mathrm{NRC}^{15}$; this is comparable to that of the present study (mean stay10 days).

The evaluation of weight for height at admission shows that $63.3 \%$ had a Z score <-3SD and $26.7 \%$ had a score of $<-4$ SD. B Kumar et al from MP found that $75.7 \%$ had a score of $<-3$ SD. Singh B et al from Uttar Pradesh reported that $89.7 \%$ had a $\mathrm{Z}$ score of , $-3 \mathrm{SD}^{16}$.

$38.3 \%$ of cases showed good weight gain (>10gm/kg/day) while $40 \%$ showed moderate gain $(5-10 \mathrm{gm} / \mathrm{kg} / \mathrm{day})$ in the study by Rinki Shah et al in Baroda ${ }^{12}$. Manish Maurya et al reported an average weight gain of $13 \mathrm{gm} / \mathrm{kg} /$ day from UP. In the present study, good weight gain was present in only $13.3 \%$ of cases while majority $(78.7 \%$ ) had moderate rate of weight gain.

\section{Limitations}

The present study does not highlight the socioeconomic factors and infant feeding practices prevalent in the study population. Follow up is a key component of successful implementation of the NRC program- the present study does not include follow up as a parameter.

\section{Conclusion}

Hospital based management of SAM children in NRC is a significant step in reducing morbidity and mortality among undernourished children. However, there is a need to improve the quality of care in terms of better weight gain, minimising drop outs and ensuring satisfactory follow up. There is a need to raise awareness among the community health care providers regarding early detection of mild to moderate malnutrition and prompt referral of severe cases to the NRC.

\section{Funding: None \\ Conflict of interest: None}

\section{Reference}

1. World Health Organization (WHO) guidelines for inpatient treatment of severely malnourished children, WHO, Geneva, Switzerland, 2003. 
2. International Institute of Population Studies and Macro international: National Family Health Survey (NFHS-3), IIPS, Mumbai, 2007.

3. $\mathrm{P}$ Ramachandran. Adoption of $\mathrm{WHO}$ growth standards (2006): Issues and implications. Bulletin of Nutrition Foundation of India.2007;23, 1-6.

4. Ann Ashworth S, Khanum, Alan Jackson, Clarie Schofield: Guidelines for the inpatient treatment of severely malnourished children; WHO guidelines Geneva, 2003; 10-48.

5. Ashworth AJ, Alan Jackson, R. Uauy : Focusing on malnutrition management to improve child survival in India; Indian Pediatrics, 2007;44(6);413-6.

6. Government of Madhya Pradesh. Manual for facility based management of severely malnourished children in Madhya Pradesh, 2011;Bhopal India: Department of Health and Family Welfare; 2011

7. M Choudhury, Deepak Sharma, Rajendra Prasad Nagar, Brahma Dutt Gupta et al: Clinical profile of Severe Acute Malnutrition in Western India: A prospective observational study from India: Journal of Pediatric and Neonatal Care; 2015;2(1): 00057.

8. Ashraf S, Javed MT, Abbas N, Aysha H et al: Malnutrition in diseased children with reference to age,sex, socioeconomic status and area of living; Int Journal Agri Biol , 2001;3(4):419-422.

9. Aneja B, Singh P, Tandon M, Pathak P et al: Etiological factors of malnutrition among infants in two urban slums of Delhi; Indian Pediatrics, 2001: 38(2); 160165.

10. Joshi s, Walgankar SS: Epidemiology of malnutrition in a rural field practice area of Navi Mumbai; Indian Journal Prev Soc $\operatorname{Med}(2004)$ - 35; 80-84
11. Bharti Bhandari, Anita Mehta, Ayesha Imran: Clinical and Anthropometric profile of Severe Acute Malnourished children on therapeutic intervention with WHO/ UNICEF recommended therapeutic food and Home based therapeutic food; International Journal of Science and Research, Vol 4, Issue 4; April 2015,268273.

12. Rinki Shah, Bakul B. Javdekar: Management of children with severe acute malnutrition: experience of nutritional rehabilitation centre at Baroda, Gujarat; International Journal of Contemporary Pediatrics; 2014 May;1(1): 3-6.

13. Bernal C, Velasquez C, Alcaraz G, Botero $\mathrm{J}$ :Treatment of severe malnutrition in children: Experience in implementing the WHO guidelines in Turbo, Columbia; Journal of Pediatric Gastroenterology and Nutrition,46(3):322-328.

14. Manish Maurya, D K Singh, Ruchi Rai, P C Mishra, Anubha Srivastava: An experience of Facility based Management of Severe Acute Malnutrition in Children aged 6-59 months adopting the World Health Organization Recommendations; Indian Pediatrics; Vol 51, June 2015, 481483.

15. K Sarala Kumari:Project ReportAssessment of weight gain in children admitted to nutritional rehabilitation centre\& afterwards for 2 year, Sangareddy, Medak, Telengana: Indian Institute of Public Health, Hyderabad: 181( accessed on 14/09/2017)

16. K Singh, N Badgaiyan, A Ranjan ,H O Dixit, A Kaushik et al: Management of children with Severe Acute Malnutrition: Experience of Nutritional Rehabilitation Centres in Uttar Pradesh, India; Indian Pediatrics, Vol 51, January 2014; 21-25. 